\title{
Propagation Protocols for Network-Wide Localization Based on Two-Way Ranging
}

Tanvir, Sadaf ; Schiller, Eryk ; Ponsard, Benoit ; Duda, Andrzej

\begin{abstract}
In this paper, we study the problem of designing propagation protocols for network-wide localization based on two-way ranging. At the beginning, a network contains a few localized anchor nodes and a large number unlocalized nodes. Unlocalized nodes in the communication range of anchor nodes perform two-way ranging, estimate their positions, and become anchor nodes. The process repeats until all nodes know their positions. We consider three protocols for this propagation process, analyze their convergence speed, and evaluate the communication costs related to the energy consumption. We show that the proposed Optimized Beacon protocol requires much less messages than two other considered protocols while achieving almost the same convergence delay as the Beacon protocol.
\end{abstract}

DOI: https://doi.org/10.1109/WCNC.2010.5506593

Posted at the Zurich Open Repository and Archive, University of Zurich

ZORA URL: https://doi.org/10.5167/uzh-174651

Conference or Workshop Item

Accepted Version

Originally published at:

Tanvir, Sadaf; Schiller, Eryk; Ponsard, Benoit; Duda, Andrzej (2010). Propagation Protocols for NetworkWide Localization Based on Two-Way Ranging. In: Networking Conference (WCNC), Sydney, 18 May 2010 - 21 May 2010. IEEE, 1-6.

DOI: https://doi.org/10.1109/WCNC.2010.5506593 


\title{
Propagation Protocols for Network-Wide Localization Based on Two-Way Ranging
}

\author{
Sadaf Tanvir, Eryk Schiller, Benoît Ponsard, Andrzej Duda \\ Grenoble Informatics Laboratory \\ Grenoble Institute of Technology, France \\ Email: \{tanvir, schiller, ponsard, duda\}@imag.fr
}

\begin{abstract}
In this paper, we study the problem of designing propagation protocols for network-wide localization based on two-way ranging. At the beginning, a network contains a few localized anchor nodes and a large number unlocalized nodes. Unlocalized nodes in the communication range of anchor nodes perform two-way ranging, estimate their positions, and become anchor nodes. The process repeats until all nodes know their positions. We consider three protocols for this propagation process, analyze their convergence speed, and evaluate the communication costs related to the energy consumption. We show that the proposed Optimized Beacon protocol requires much less messages than two other considered protocols while achieving almost the same convergence delay as the Beacon protocol.
\end{abstract}

Index Terms-Wireless Sensor and Actuator Networks, network-wide localization, two-way ranging

\section{INTRODUCTION}

We consider the problem of localization in wireless multihop networks. In such networks, the information about the node location is an important feature, because it opens new opportunities to user applications, in-network information processing algorithms, or geographic routing protocols. In Wireless Sensor and Actuator Networks (SANET), location information is crucial for correct interpretation of measured data. SANETs are composed of small, low-cost, sensor/actuator nodes with radio communication capacity. They can have hundreds to thousands of nodes deployed outdoor or indoor; getting the location of nodes across the whole network is thus a real challenge in both theoretical and practical perspective. One of the important aspects of supporting localization is energy efficiency-we want to achieve network-wide localization at the smallest drained energy possible.

SANET nodes can use GPS (Global Positioning System) for localization [1], however this technology presents several drawbacks: significant cost, energy consumption, and limitation to outdoor deployment. A more practical approach is to use wireless communication to measure distances between nodes and derive geographical positions with respect to a small number of anchor nodes (AN) with known coordinates. Several authors proposed various localization techniques [2], [3], [4].

Tri- or multilateration techniques have recently attracted an increased interest because of the progress in low-power implementation of two-way ranging (TWR) using ultra-wide band (UWB) communications. Several authors reported on its feasibility [5], [6], [7] and a recent addendum to the IEEE 802.15.4 standard [8], [9] includes this function.

Two-way ranging and tri- or multilateration enable networkwide localization in which the network starts with a limited number of anchor nodes that serve as references to other unlocalized nodes (UNs). If an unlocalized node can estimate its distance to three or more anchor nodes, it determines its position through trilateration and becomes an anchor node. Nodes can further refine their positions to achieve a desired level of accuracy [10]. The process spreads all over the network and repeats until all nodes becomes anchors.

In this paper, we consider the problem of designing protocols for network-wide propagation of localization based on two-way ranging. We consider an existing protocol and propose its two refinements for node cooperation leading to progressive position estimation of all nodes in the network. We compare the protocols with respect to the speed of convergence and communication cost, which is the major part of the energy spent in localization.

The rest of the paper is organized as follows. We discuss ranging techniques in Section II and consider three networkwide propagation protocols in Section III. We report on simulation evaluation of the proposed protocols in Section IV. Section V briefly presents the related work and Section VI concludes the paper.

\section{RANGING TECHNIQUES}

Localization techniques rely on various techniques for measuring the distance between two nodes: Time of Arrival (TOA), Time Difference of Arrival (TDOA), Received Signal Strength indicator (RSS), and Two-Way Ranging (TWR) [11]. Among these methods, two-way ranging is the most interesting one for low cost and energy constrained sensor nodes since it does not require neither accurate clock synchronization nor additional hardware. Several authors reported on its suitability for sensor networks and practical deployment issues [12], [13].

Two-way ranging consists in measuring the round-trip time of signal transmission between two nodes. Its accuracy strongly depends on precise recording of emission and reception instants at the physical layer. The MAC layer controls the process by triggering the emission of ranging frames [9], [14]. Figure 1 illustrates how Node A can estimate the distance based on several timestamps of transmission/reception instants: 


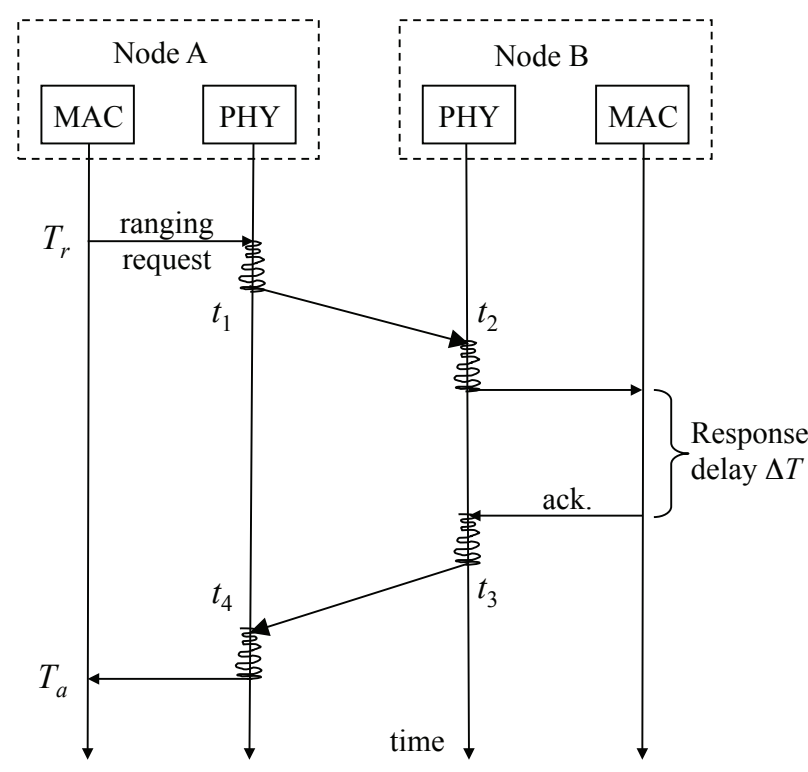

Fig. 1. The principle of two-way ranging.

$$
D_{A, B}=v \cdot \frac{t_{4}-t_{1}-\left(t_{3}-t_{2}\right)}{2}
$$

where $v$ is the propagation speed of the signal (the speed of light for radio signals), $t_{1}$ and $t_{3}$ (resp. $t_{2}$ and $t_{4}$ ) are the emission instants (resp. reception instants) of ranging frames. Since the expression only involves time differences that depend on local clocks, the technique does not require clock synchronization. Nevertheless, the technique may suffer from some limitations due to several issues: i) clock drift in cheap sensor nodes, ii) different response delays of nodes [13], iii) channel impairment such as multi-path transmission and multi-user interference [14]. In the rest of the paper, we however neglect all these issues and focus on protocols for location propagation.

\section{Protocols FOR LOCATION PROPAGATION}

We assume that sensor nodes measure distances by means of two-way ranging and we consider the problem of propagating the localization information in large scale sensor networks (typically with more than 1000 nodes). In such a network, not all nodes are in the communication range of three or more anchor nodes, so that nodes need to cooperate in the location propagation: unlocalized nodes in the communication range of anchor nodes can perform ranging, estimate their positions, and become anchor nodes. Then they can support localization of other unlocalized nodes in their neighborhood. The process repeats until all nodes know their positions.

There are several possibilities of organizing the cooperation between nodes during the location propagation process. We consider one existing protocol for this process and propose its two refinements. We analyze their convergence conditions and speed to achieve network-wide localization and evaluate the communication cost related to energy consumption.
We consider a large scale random 2D sensor network with three non-collinear anchor nodes deployed in the center of the network that we call anchor nuclei. We assume that they have overlapping communication ranges.

\section{A. Beacon Protocol}

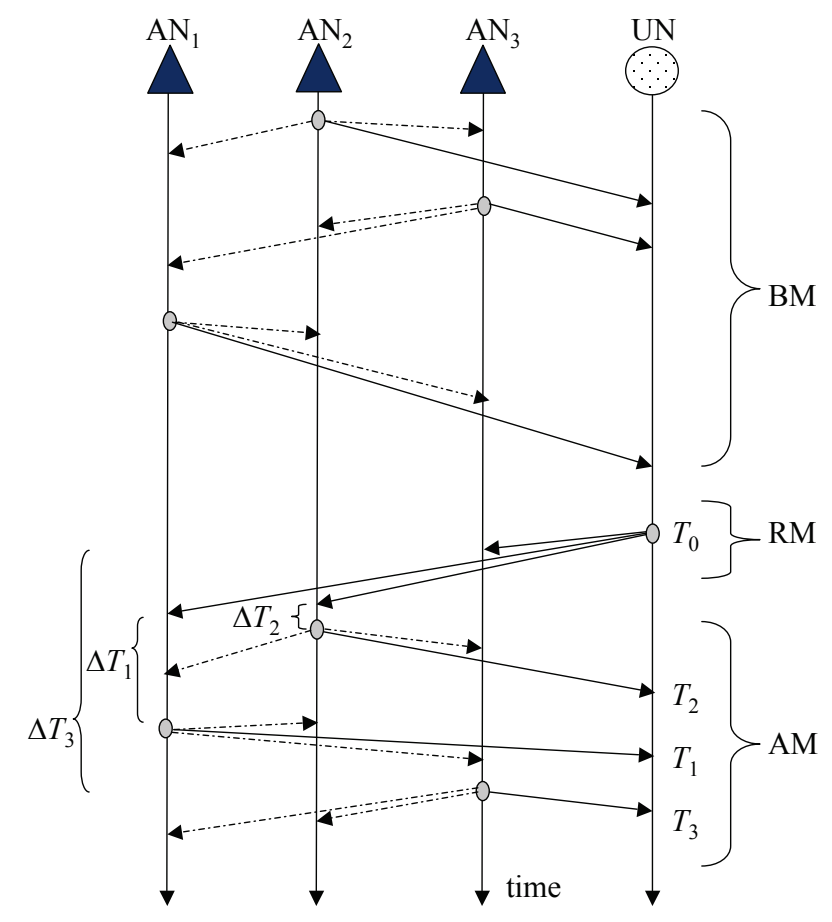

Fig. 2. The principle of the Beacon protocol.

In the Beacon protocol similar to the technique of Savvides et al. [4], anchor nodes initiate the localization process by periodically sending broadcast beacon messages (BM) each time interval $T_{B}$. When an unlocalized node receives three beacons from at least three different anchor nodes, it waits for a random uniformly distributed interval between 0 and $T_{R}$ and sends a broadcast range message (RM) to perform ranging with the anchor nodes in parallel. We introduce an additional random delay at the receivers to reduce collisions: the anchor nodes reply to the RM message with unicast $A C K$ messages (AM) after $\Delta T$, a uniformly distributed interval between 0 and $T_{A}$ to avoid simultaneous arrivals at the unlocalized node. When the anchor node schedules an ACK message to send after the interval, it may receive other ranging messages before the interval expires. In this case, the node sends multiple ACK messages just after the end of the time interval. This procedure shortens convergence latency. Timestamping the range and the ACK messages enable the estimation of the distance to three anchor nodes yielding thus the position. The unlocalized node then becomes an anchor node and starts sending periodic beacons to propagate the localization process to other unlocalized nodes. Figure 2 illustrates the principle of the Beacon protocol. 


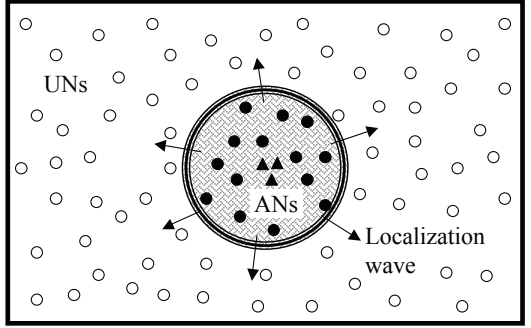

Fig. 3. Propagation of the localization wave under the Beacon protocol: the grey area contains anchor nodes that send beacons.

If we look at the way the location process propagates, we can observe a localization wave that originates at the center of the network and progresses towards the network boundaries (cf. Figure 3). When all the nodes become localized, they continue to send beacon messages. Nodes can detect the end of the location propagation by observing that there are no more range messages during a time interval. However, if nodes do not send beacons after the end of the process, there is no way of providing localization to newly added nodes.

\section{B. Continuous-Ranging Protocol}

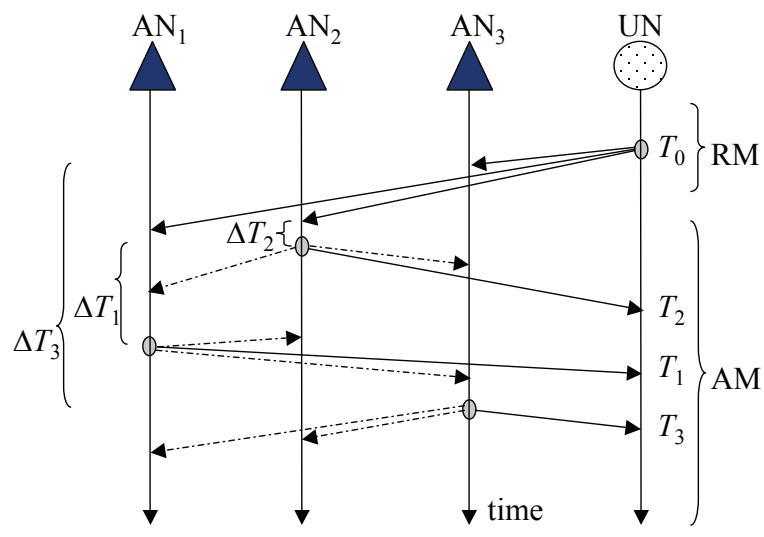

Fig. 4. The principle of the Continuous-Ranging protocol.

In the Continuous-Ranging protocol, unlocalized nodes initiate localization by periodically sending broadcast range messages each time interval $T_{B}$. The propagation process starts at the center of the network with anchor nodes replying to the range messages with unicast ACK messages after $\Delta T$, a random uniformly distributed interval between 0 and $T_{A}$. The anchor nodes also may send multiple ACK messages after the the time interval if needed. When the unlocalized node receives three ACK messages from three different anchor nodes, it estimates its position through trilateration, becomes an anchor node and stops sending range messages while listening to range messages from other nodes. Figure 4 illustrates the principle of the Continuous-Ranging protocol.

When observing the localization process at the macroscopic level, we can notice similar propagation of the localization

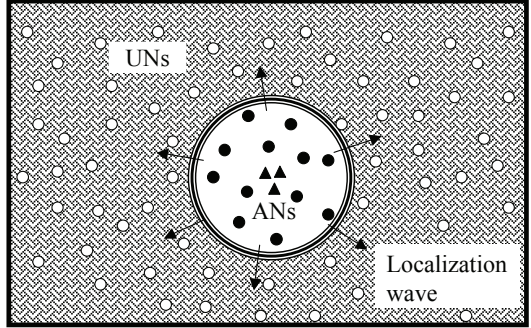

Fig. 5. Propagation of the localization wave under the Continuous-Ranging protocol: the grey area contains unlocalized nodes that send range messages.

wave: nodes close to nuclei start ranging and the wave propagates towards edges (cf. Figure 5). Once all nodes become localized, the process stops and nodes do not send any localization messages. Adding a new unlocalized node is still possible, because it is up to the new node to start its ranging process. The drawback of this approach is that unlocalized nodes farther from the center of the network send their range messages even if the localization wave has not yet arrived in their neighborhood.

\section{Optimized Beacon Protocol}

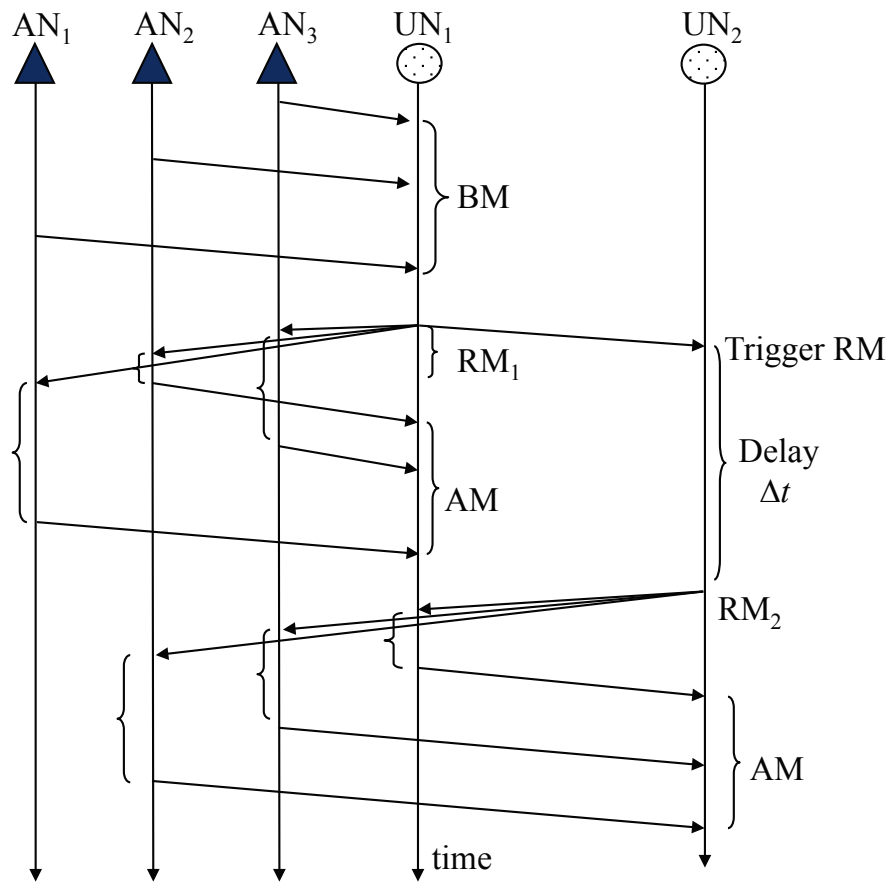

Fig. 6. The principle of the Optimized Beacon protocol.

In the Optimized Beacon protocol, we try to reduce the number of messages. Like in the Beacon protocol, anchor nuclei initiate the localization process by periodically sending a limited number of broadcast beacon messages so that any unlocalized node in their neighborhood can start ranging. However, the difference is that once an unlocalized node gets localized, it does not send beacons. Unlocalized nodes sense 
ranging packets in order to start their ranging operations. We assume that if an unlocalized node overhears three broadcast range messages sent by other nodes, it can start its ranging operation without waiting for beacons (cf. Figure 6). It waits however for random interval $\Delta t$ composed of two parts: first it waits for constant interval $T_{A}$ to be sure that when its neighboring nodes have started the ranging operation, they finished it so they are localized and ready to respond with AM messages. Then it waits for a random uniformly distributed interval between 0 and $T_{R}$ to avoid simultaneous transmissions. Delays $T_{A}, T_{R}$, and the ACK generation procedure are the same as in the previous schemes. Under this protocol, the localization wave still goes from the center of the network to its boundaries, but nodes send range messages only in the vicinity of the wave (cf. Figure 7).

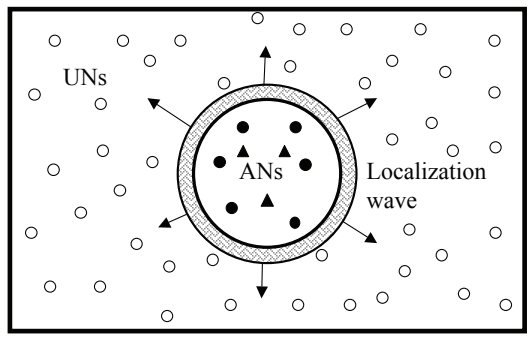

Fig. 7. Propagation of the localization wave under the Optimized Beacon protocol: the grey area contains nodes that send range messages.

\section{Evaluation}

In this section, we evaluate the proposed protocols through simulations and study their convergence as well as energy consumption related to communication costs. We use ns -2 to run simulations [15].

We randomly place $N$ sensor nodes in a square of size $L \mathrm{x} L$ $\mathrm{m}^{2}$ according to the uniform distribution. We use the Two Ray Ground propagation model for the radio communication range and we assume the IEEE 802.11b PHY-MAC layers. The mean number of neighbors per node $\eta$ (also called the average network degree) is then proportional to the mean node density defined by $\rho=N / L^{2}$, transmission power, and receiving threshold. We set configuration parameters of the protocols described above to the following values: $T_{B}=20 \mathrm{~s}, T_{R}=3$ $\mathrm{s}$, and $T_{A}=3 \mathrm{~s}$.

\section{A. Convergence}

First of all, we analyze the convergence of the propagation process in function of the average node degree $\eta$ that we vary by adjusting transmission power of nodes. Figure 8 shows the relationship between $\eta$ and the proportion of localized nodes in the network after the propagation process. We can observe that the network needs to have some critical node density so that each node finds three localized neighbors during the process and obtains its position.

We compare the three curves with a theoretical transition curve $G_{3}(1350, \eta)$ (cf. Figure 8 ) in a network with 1350 nodes

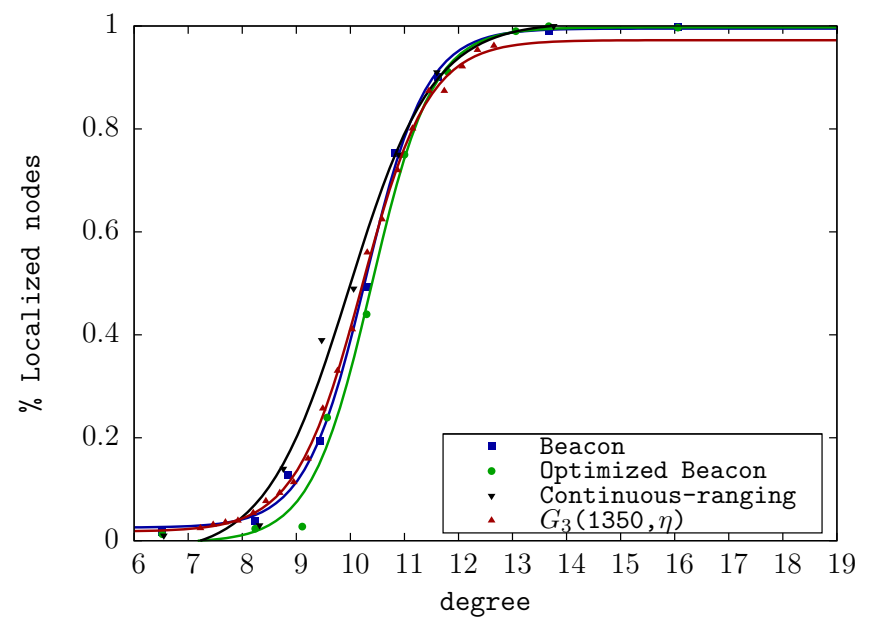

Fig. 8. Critical node degree for the three protocols, $N=1350$ nodes, $L=300 \mathrm{~m}$.

operating according to the 3-propagation process [16]. In a $k$-propagation process, the network initially contains white nodes and a node becomes black if it receives a token from its $k$ neighbors. Flooding is an example of a 1-propagation process. We can see that all the curves for the propagation protocols perfectly match the theoretical curve. This shows that they behave like a percolation processes in a finite network [17].

\section{B. Convergence Latency}

In this simulation, we record the delay for nodes to become localized and plot them against the distance from the anchor nuclei (we average data over all nodes located at same distance from the center). The network parameters are the following: $L=300 \mathrm{~m}, N=1350, \eta=13.5$. Figure 9 presents the convergence latency of the three protocols.



Fig. 9. Convergence latency: propagation delay experienced by nodes versus their distance from the anchor nuclei.

We can observe that the Beacon protocol achieves the shortest convergence delay and its optimized version converges 
almost in the same time only after a slightly longer delay. The two protocols propagate the localization information across the network with an almost constant linear speed. The ContinuousRanging protocol performs much worse by taking more than twice time to converge.

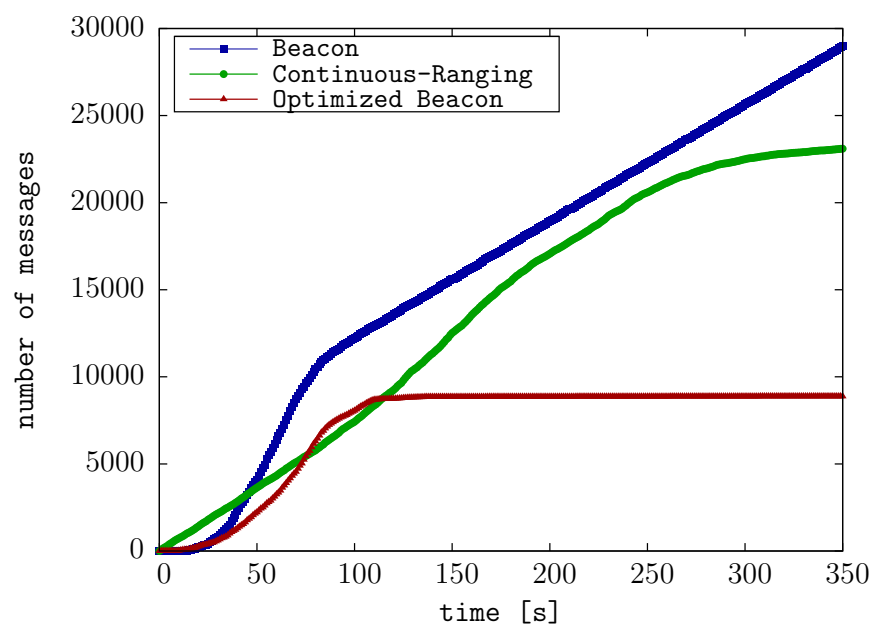

Fig. 10. Total number of messages for the three protocols.

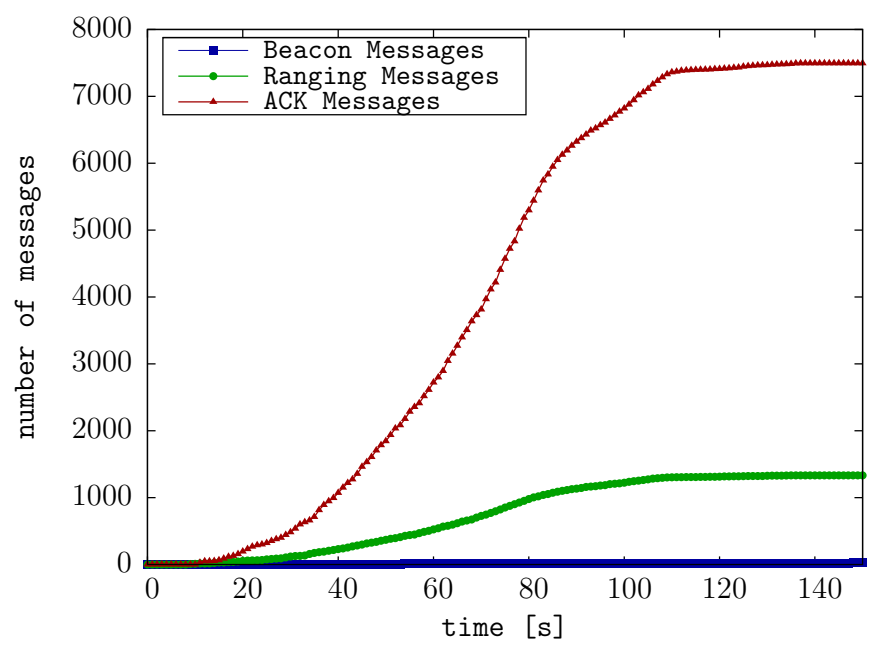

Fig. 11. Number of messages for the Optimized Beacon protocol.

\section{Communication Costs}

We consider here communication costs as the most important factors that influence energy consumption. We adopt a simple energy model in which energy consumption is proportional to the number of transmitted and received messages. We are aware that such a model is only a rough approximation, because it does not take into account more sophisticated node operation with different energy levels at various node states, but our goal in this paper is a first order analysis of protocols and their comparison. Moreover, we assume a WLAN MAC layer not oriented towards energy savings and a more complex energy model would also require considering different sensor network MAC layers such as WiseMAC [19] or SCP [20], which we plan to take into account in the future work. Thus, we compare three proposed protocols with respect to the number of transmitted and received messages during the localization and propagation processes.

Figure 10 shows the number of messages for a network with $L=300 \mathrm{~m}$ and $N=1350$ nodes with the mean node degree $\eta=13.5$. The Beacon and Continuous-Ranging protocols result in a large number of messages mainly due to continuous transmissions of beacon and ranging messages. Moreover, the Beacon protocol continues to generate messages after convergence. The Optimized Beacon protocol largely reduces the number of messages required for localization and the process of sending ranging messages terminates when the network is localized. Figure 11 presents the number of messages for the Optimized Beacon protocol. Most of them are ranging and ACK messages that are required for localization. The number of beacon messages remains very small.

\section{RELATED WORK}

We limit this short section to the work strictly relevant to our paper, because the literature is abundant. Many authors considered different techniques required for localization: Time of Arrival (TOA) [11], Angle of Arrival (AoA) [21], Time Difference of Arrival (TDOA) [3], [4], [22], Received Signal Strength indicator (RSS) [23] and RSS profiling [24]. Many of them are not suitable for wireless sensor networks [25]: experiments showed that the AOA method is practically unusable on sensor nodes, the TDOA technique requires highly directional, expensive, and energy consuming ultrasonic transducers, while the propagation speed of sound depends on external factors like temperature and humidity, the RSS technique is a poor range measurement technique, and the one way measurements of the signal propagation time requires synchronized clocks, which is difficult to obtain on energy constrained and cheap sensor nodes.

Recent advancements in the ultra-wide band technology and chirp transmission [26] makes the roundtrip propagation time measurement a feasible technique for ranging in wireless sensor networks [14], [12], [13]. Two-way ranging presents several advantages [5]: it does not require additional hardware nor synchronized clocks and gives reliable estimates [6]. Moreover, it becomes practically deployable with its specification in the 802.15.4 standard [8], [9].

Once we have a possibility of measuring distance between two nodes, a node can determine its position with respect to the positions of the other nodes through tri- or multi-lateration. Then, it needs to propagate the localization information in the network so that all nodes become localized. Some authors proposed cooperation protocols for network-wide localization. Savvides et al. proposed an iterative beacon nodes based protocol using ultrasonic ranging [4]. Nawaz et al. defined a cooperative protocol to localize the entire network in a local coordinate system with virtual coordinates. Shin et al. introduced a cooperative localization method that symmetrically performs multiway ranging in two phases to improve 
accuracy of localization [7]. The Beacon protocol proposed in this paper is inspired by the technique of Savvides et al. [4] whereas Continuous-ranging and Optimized Beacon protocols are tuned to reduce the number of exchanged messages.

\section{CONCLUSIONS}

In this paper, we have considered the problem of designing protocols for network-wide propagation of location based on two-way ranging. We have considered three protocols for node cooperation leading to progressive position estimation of all nodes in the network. We have compared the protocols with respect to the speed of convergence and communication costs.

Our simulation results first show that the three propagation protocols require sufficient network density: the mean node degree needs to be above a certain critical value depending on the 3-propagation process, so that the network attains a percolation state. The Beacon protocol achieves the shortest convergence delay, but its optimized version also converges fast and only terminates after a slightly longer delay. We also estimate the energy spent in localization through a simple model based on communication costs. We observe that the Optimized Beacon protocol requires much less messages than two other protocols.

The proposed protocols propagate the position information through the network. As each position estimation with respect to three neighbors results in an error, the localization accuracy progressively becomes worse due to error accumulation. We plan to investigate this problem and find suitable position refinement techniques to limit this effect.

\section{ACKNOWLEDGMENTS}

This work was partially supported by the French National Research Agency (ANR) project ARESA under contract ANR05-RNRT-01703 and Higher Education Commission (HEC) of Pakistan.

\section{REFERENCES}

[1] B. Parkinson and J. Spilker Jr, The Global Positioning System: Theory and Applications, vol. 1 and 2. American Institute of Aeronautics and Astronautics, 1996.

[2] C. Savarese, J. M. Rabaey, and K. Langendoen, "Robust Positioning Algorithms for Distributed Ad-Hoc Wireless Sensor Networks," in Proc. USENIX Annual Technical Conference, pp. 317-327, 2002.

[3] A. Savvides, C.-C. Han, and M. B. Strivastava, "Dynamic Fine-Grained Localization in Ad-Hoc Networks of Sensors," in Proc. MobiCom 2001, (Rome, Italy), pp. 166-179, 2001.

[4] A. Savvides, H. Park, and M. B. Srivastava, "The N-hop Multilateration Primitive For Node Localization Problems," Mobile Networks and Applications Journal, vol. 8, number 4, pp. 443-451, August 2003.
[5] S. Lanzisera, D. T. Lin, and K. S. Pister, "RF Time of Flight Ranging for Wireless Sensor Network Localization," in Proc. of WISES 2006, (Vienna, Austria), June 2006.

[6] T. Karalar and J. Rabaey, "An RF ToF Based Ranging Implementation for Sensor Networks," in Proc. IEEE ICC 2006, vol. 7, (Istanbul, Turkey), pp. 3347-3352, June 2006.

[7] F. Shin, L. Zhiwei, and L. J. Xing, "Symmetric Multi-Way Ranging for Wireless Sensor Network," in Proc. IEEE PIMRC 2007, (Athens, Greece), pp. 1-5, September 2007.

[8] IEEE 802.15.4a-2007. http://standards.ieee.org.

[9] Z. Sahinoglu and S. Gezici, "Ranging in the IEEE 802.15.4a Standard," in Proc. of IEEE WAMICON'06, pp. 1-5, December 2006.

[10] J. Liu, Y. Zhang, and F. Zao, "Robust Distributed Node Localization with Error Management," in Proc. ACM MobiHoc 2006, (Florence, Italy), pp. 250-261, May 2006.

[11] G. Mao, B. Fidan, and B. D. O. Anderson, "Wireless Sensor Network Localization Techniques," Computer Networks, vol. 51, pp. 2529-2553, July 2007.

[12] R. Hach, "Symmetric Double Sided Two-Way Ranging." IEEE 802.15.4a Ranging Subcommittee, June 2005.

[13] L. J. Xing, L. Zhiwei, and F. Shin, "Symmetric Double Side Two Way Ranging with Unequal Reply Time," in Proceedings of Vehicular Technology Conference (VTC'07 Fall), (Baltimore, MD, USA), pp. 19801983, 30 September - 03 October 2007.

[14] J. Decuir, "Two Way Time Transfer Based Ranging." Contribution to the IEEE 802.15.4a Ranging Subcommittee, October 2004.

[15] "The Network Simulator ns-2." http://www.isi.edu/nsnam/ns/.

[16] E. Schiller, S. Tanvir, B. Ponsard, and A. Duda, "Percolation of kpropagation in wireless multi-hop networks," in submitted to INFOCOM 2010.

[17] J. Dall and M. Christensen, "Random geometric graphs," Phys. Rev. E vol. 66, Jul 2002.

[18] J. Polastre, J. Hill and D. Culler, "Versatile Low Power Media Access for Wireless Sensor Networks," In Proceedings of ACM SenSys, 2004.

[19] C. Enz, A. El-Hoiydi, J. Decotignie, V. Peiris., "WiseNET: An UltralowPower Wireless Sensor Network Solution," IEEE Computer, vol. 37, pp. 62-70, August 2004.

[20] W. Ye, F. Silva and J. Heidemann, "Ultra-Low Duty Cycle MAC with Scheduled Channel Polling," In Proceedings of ACM SenSys, Boulder, CO, November 2006.

[21] D. Niculescu and B. Nath, "Ad hoc Positioning System (APS) using AOA," in Proceedings of INFOCOM 2003, vol. 3, pp. 1734-1743, April 2003.

[22] S. Nawaz and S. Jha, "Collaborative Localization for Wireless Sensor Networks," in Proceedings of the 18th Annual IEEE International Symposium on Personal, Indoor and Mobile Radio Communications (PIMRC07), Sept. 2007.

[23] P. Bergamo and G. Mazzini, "Localization in Sensor Networks with Fading and Mobility," in Proceedings of 13th IEEE International Symposium on Personal, Indoor and Mobile Radio Communications(PIMRC02), vol. 2, pp. 750-754, Sept. 2002.

[24] P. Bahl and V. N. Padmanabhan, "RADAR: An In-building RF-based User Location and Tracking System," in Proceedings of IEEE INFOCOM 2000, vol. 2, (Tel-Aviv, Israel), pp. 775-784, 2000.

[25] H. Karl and A. Willig, Protocols and Architectures for Wireless Sensor Networks. John Wiley and Sons, Ltd, 2006.

[26] R. Hach, J. Lampe, and L. Menzer, "DBO-CSS PHY Presentation for 802.15.4a." Contribution to the IEEE P802.15 Working Group for Wireless Personal Area Networks(WPANs), March 2005. 